\title{
Infecção experimental por Mycoplasma gallisepticum e Escherichia coli em perus ${ }^{1}$
}

\author{
Maria E. Moraes ${ }^{2}$, Gabriele B.A. Pereira² ${ }^{2}$ Claudete S. Astolfi-Ferreira ${ }^{2}$ \\ e Antonio J. Piantino Ferreira ${ }^{2 *}$
}

\begin{abstract}
Moraes M.E., Pereira G.B.A., Astolfi-Ferreira C.S. \& Ferreira A.J.P. 2013. [Experimental infection with Mycoplasma gallisepticum and Escherichia coli in turkeys.] Infecção experimental por Mycoplasma gallisepticum e Escherichia coli em perus. Pesquisa Veterinária Brasileira 33(8):975-978. Setor de Ornitopatologia, Departamento de Patologia, Faculdade de Medicina Veterinária e Zootecnia, Universidade de São Paulo, Avenida Prof. Dr. Orlando Marques de Paiva 87, Cidade Universitária, São Paulo, SP 05508-000, Brazil. E-mail: ajpferr@usp.br

Mycoplasma gallisepticum (MG) causes infectious sinusitis in turkeys, and is commonly associated with Escherichia coli. The objective of this study was to develop in turkeys an experimental model for infectious sinusitis. Two hundred and fifty male turkeys of Nicholas breed (Aviagen ${ }^{\circledR}$ ) were divided into negative control group and challenged, animals were housed until 42 days old. The birds were inoculated in the first day of age with the MG vaccine (F-VAX ${ }^{\circledR}$ Schering Plough) and on day 21 with E. coli. We analyzed the mortality, clinical signs and lesions in air sacs, liver and heart. The results showed that the vaccine against Mycoplasma gallisepticum (MG-F) is pathogenic for turkeys and that the experiment was able to simulate natural infection with MG and E. coli.
\end{abstract}

INDEX TERMS: Mycoplasma gallisepticum, Escherichia coli, turkeys, infectious sinusitis, experimental infection.

RESUMO.- Mycoplasma gallisepticum (MG) é responsável por provocar sinusite infecciosa em perus. A infecção por Mycoplasma spp. torna a ave susceptível a infecção por Escherichia coli. $\mathrm{O}$ objetivo deste estudo foi desenvolver em perus, um modelo experimental para a sinusite infecciosa. Utilizou-se 250 peru,s machos da linhagem Nicholas (Aviagen $囚$ ) divididos em grupo não infectado (T1) e grupo desafiado (T2) que recebeu por via ocular, com um dia de idade, Mycoplasma gallisepticum cepa F e aos 21 dias de idade E. coli por via saco aéreo. Analisou-se a mortalidade, os sinais clínicos e lesões em sacos aéreos, fígado e coração. Concluiu-se que o delineamento experimental utilizado foi eficaz para simular a infecção natural por MG e E. coli, sendo que a vacina contra MG-F utilizada para poedeiras é patogênica para perus.

TERMOS DE INDEXAÇÃO: Mycoplasma gallisepticum, Escherichia coli, perus, sinusite infecciosa, infecção experimental.

\footnotetext{
${ }^{1}$ Recebido em 26 de setembro de 2012.

Aceito para publicação em 31 de maio de 2013.

${ }^{2}$ Departamento de Patologia, Faculdade de Medicina Veterinária e Zootecnia (FMVZ), Universidade de São Paulo (USP), Av. Prof. Dr. Orlando Marques de Paiva 87, Cidade Universitária, São Paulo, SP 05508-000, Brasil. *Autor para correspondência: ajpferr@usp.br
}

\section{INTRODUÇÃO}

Mycoplasma gallisepticum (MG) é uma bactéria que pertence ao gênero Mycoplasma. Provoca sinusite infecciosa em perus e doença respiratória crônica em frangos, sendo que nos perus a doença apresenta um quadro infeccioso mais grave do que em frangos. Os sinais clínicos normalmente observados são estertores, secreção nasal, espirros, conjuntivite e em perus é frequente a sinusite infraorbital (Ley 2008). A infecção por MG aumenta a mortalidade, a condenação de carcaça e a conversão alimentar, além disso, reduz a produção de ovos e a eclodibilidade (Evans et al. 2009).

Mycoplasma gallisepticum é um patógeno de grande relevância para aves, em consequência de sua alta transmissão. Dentre as espécies de micoplasma que acometem as galinhas e os perus, MG é o de maior importância econômica (Evans et al. 2005).

Buim et al. (2009) analisaram 1046 amostras de suabes de traquéia e embriões bicados oriundas de diferentes estabelecimentos de avicultura comercial dos estados de São Paulo, Paraná e Pernambuco, que apresentavam problemas respiratórios ou queda na produção de ovos. A prevalência de M. gallisepticum e M. synoviae (MS) foi de $72,7 \%$, o que comprova a alta disseminação de Mycoplasma spp. nas 
granjas avaliadas, sendo que a incidência de MS foi maior do que a de MG.

Uma das vacinas utilizadas para a proteção contra MG éa vacina viva, da cepa F, pois estimula uma boa proteção contra desafios por MG, reduz as perdas na produção de ovos, contudo o seu uso é limitado devido a sua patogenicidade para perus e frangos de corte jovens (Evans et al. 2009).

A infecção por micoplasma geralmente está associada a outros patógenos respiratórios como vírus ou Escherichia coli (Kleven 1998). As aves saudáveis são pouco susceptíveis a infecções por E. coli quando em exposições naturais, independentemente das características de virulência da bactéria. As infecções por E. coli, em aves, se apresentam de forma sistêmica, principalmente quando existe imunossupressão como consequência de infecções bacterianas, virais, estresse ou de condições ambientais inadequadas, como por exemplo a ventilação deficiente do plantel (Barnes et al. 2008).

Escherichia coli é um patógeno que acomete aves de produção em todo o mundo, pois as alterações no trato respiratório de frangos ou de perus oferecem um ambiente favorável para que E. coli se instale (Glisson 1998), assim pode-se considerar que trata-se de um agente comum em doenças respiratórias nos perus (Garmyn 2009). A infecção por Mycoplasma spp. torna a ave susceptível a E. coli, sendo que esses patógenos podem levar a quadros de aerossaculite grave (Ley 2008).

O objetivo deste trabalho foi desenvolver em perus, um modelo experimental para a sinusite infecciosa.

\section{MATERIAL E MÉTODOS}

Foram alojados do primeiro ao $42^{\circ}$ dia de idade, 250 perus machos da linhagem Nicholas (Aviagen ${ }^{\circledR}$ ) e divididos em dois tratamentos, sendo que cada tratamento foi subdividido em cinco repetições com 25 perus que foram mantidos em boxes de $1,7 \mathrm{~m}^{2}$, e receberam água e ração ad libitum.

0 tratamento não infectado com a vacina contra MG e Escherichia coli foi denominado tratamento 1 (T1-controle negativo) e o tratamento 2 (T2-grupo infectado), foi inoculado com um dia de idade, por via ocular, com a vacina contra Mycoplasma gallisepticum (F-VAX Schering Plough ${ }^{\circledR}$ ) na concentração preconizada para galinhas poedeiras. Aos 21 dias de idade o tratamento T2 foi inoculado pela via do saco aéreo torácico esquerdo com $0,1 \mathrm{~mL}$ de E. coli $\left(1 \times 10^{7} \mathrm{UFC} / \mathrm{mL}\right.$ ). A cepa utilizada foi E. coli (EC 341, sorogrupo 0119) pertencente à coleção de cultura do Laboratório de Ornitopatologia da FMVZ-USP.

A mortalidade foi observada duas vezes ao dia, durante todo o experimento e as aves foram avaliadas com relação aos sinais clínicos como: apatia, sonolência, espirros, edema facial, secreção ocular, secreção nasal e estertores. As aves que morreram após o desafio com E. coli foram necropsiadas para a observação de lesões no saco aéreo abdominal, saco aéreo torácico esquerdo e direito, além de possível comprometimento do fígado (perihepatite) e coração (pericardite). Após uma semana do desafio com $E$. coli (28 dias de idade) e aos 42 dias de idade, foram sacrificadas respectivamente 80 e 90 aves, para que fossem observadas lesões como as descritas acima e também foi colhido sangue para a realização de exames sorológicos pela técnica de Inibição da Hemaglutinação (HI) para a detecção de anticorpos para MG, sendo que no dia 28 foi colhido o sangue de todas as aves sacrificadas e no dia 43 foi colhido apenas de 5 (cinco) aves de cada repetição.
O experimento foi aprovado pela "Comissão de Ética no uso de animais" da Faculdade de Medicina Veterinária e Zootecnia da Universidade de São Paulo, sob o protocolo 2651/2012.

\section{RESULTADOS}

Aos 10 dias de experimento, no tratamento T2, o primeiro sinal clínico observado foi espirro. No $12^{\circ}$ dia foi observado no tratamento desafiado (T2), edema facial infraorbital e no $14^{\circ}$ dia e secreção nasal. A secreção ocular passou a ser observada no $17^{\circ}$ dia e no $33^{\circ}$ dia observaram-se estertores traqueais. Esses sinais clínicos estão ilustrados nas Figuras 1 e 2. Além desses sinais durante o experimento, três aves apresentaram sinais neurológicos como desequilíbrio, perda da propriocepção e tremores, sendo que uma ave apresentava torcicolo e opistótono.

Após a infecção com Escherichia coli, entre o 22으 e o $42^{\circ}$ 을 dia, 60 aves morreram (48\%) no grupo desafiado (T2), e enquanto no tratamento controle (T1) apenas duas $(n=2)$ aves $(1,6 \%)$ morreram.É importante ressaltar que 53 aves do grupo desafiado (T2) morreram nas primeiras 48 horas após o desafio com E. coli, ou seja, $42,4 \%$ do total de aves desse grupo.

Nas necropsias, após a inoculação com E. coli no grupo

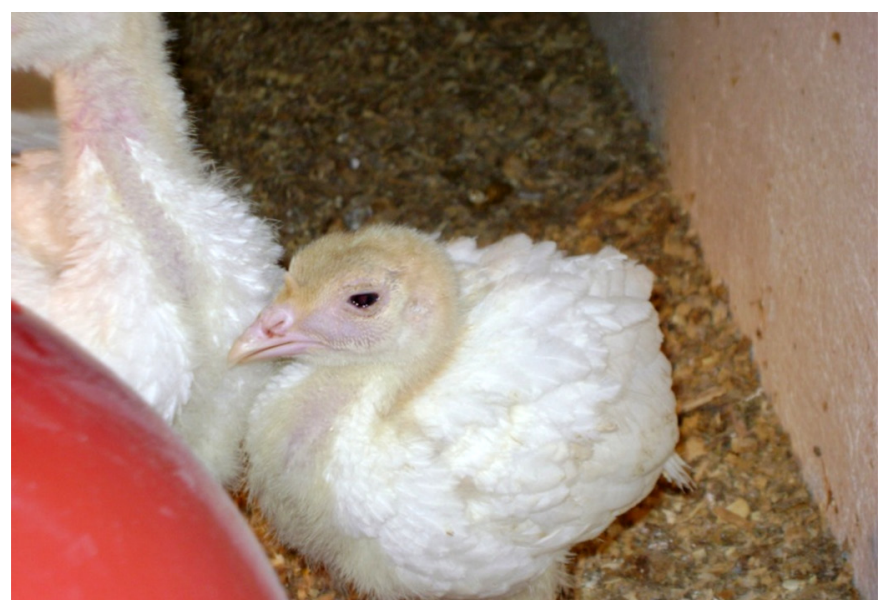

Fig.1. Ave do tratamento desafiado com Escherichia coli (T2) apresentando edema facial infraorbital e secreção ocular.

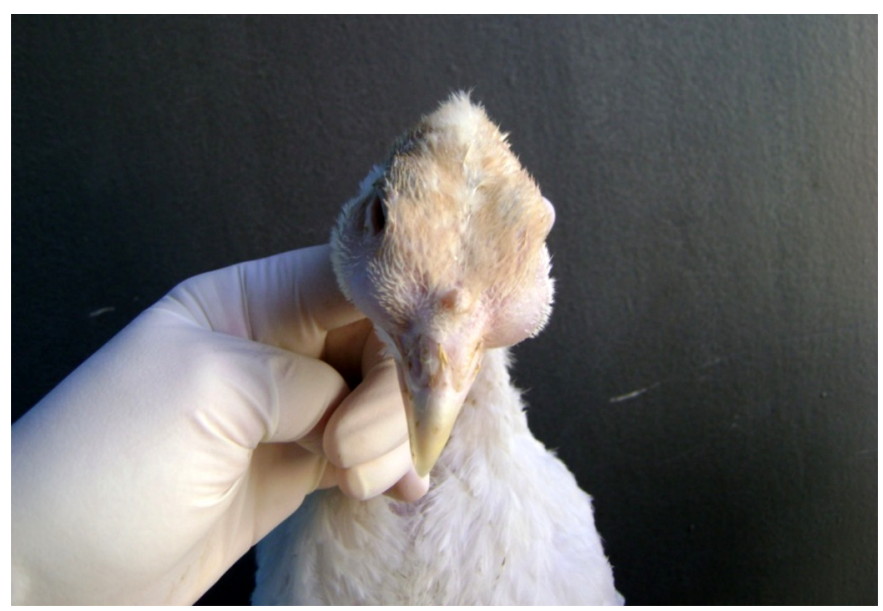

Fig.2. Ave do tratamento desafiado com Escherichia coli (T2) com edema facial infraorbital bilateral. 


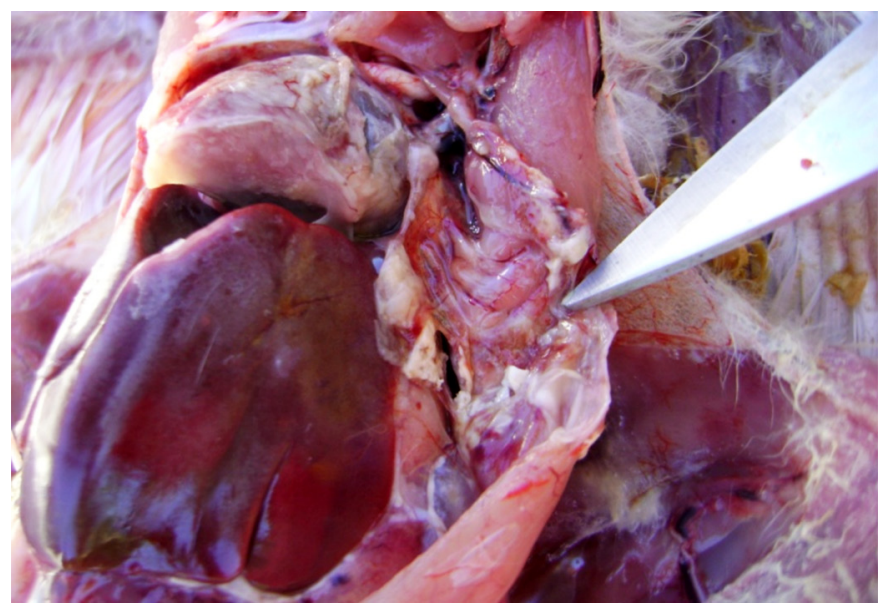

Fig.3. Ave do tratamento desafiado com Escherichia coli (T2) com pericardite e aerossaculite em saco aéreo torácico esquerdo.

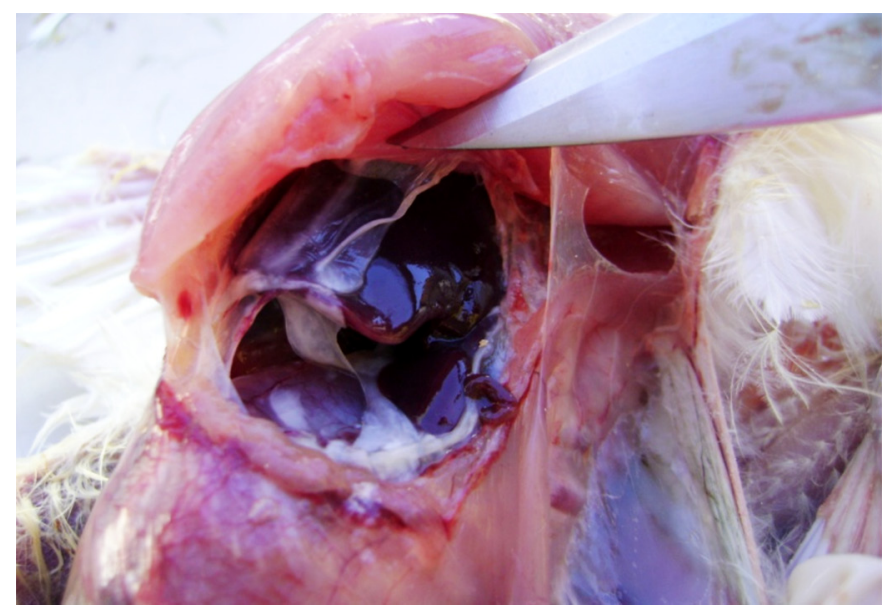

Fig.4. Ave do tratamento desafiado com Escherichia coli (T2) com perihepatite.

T2 foram observadas lesões como perihepatite, pericardite e aerossaculite. Essas lesões são mostradas nas Figuras 3 e 4. Após três semanas da infecção ocorreram lesões em sacos aéreos em maior proporção do que uma semana após o desafio, contudo houve redução nas lesões de fígado e coração. 0 Quadro 1 mostra a porcentagem de aves necropsiadas do grupo desafiado (T2) com algum grau de lesão após a infecção com E. coli nos dois períodos estudados. As aves do tratamento controle não constam no quadro, pois não havia lesões nestas aves.

Os resultados de HI para MG no tratamento controle (T1) no primeiro e no segundo período (28 e 42 dias) foram de títulos abaixo de 1:20, considerados negativos para a espécie estudada. No grupo T2, 28 aves foram positivas (média dos títulos 1:602/ ou títulos acima de 1:80 que são considerados positivos para a espécie/ ou títulos variando entre $1: 80$ a 1:1280)e no primeiro sacrifício (93,33\%). No segundo sacrifício 18 aves se mostraram positivas para MG, ou seja, com títulos acima de 1:80 (média de títulos 1:230). 0 Quadro 3 mostra a porcentagem de aves positivas no teste de Inibição da Hemaglutinação (HI) para Mycoplasma gallisepticum no primeiro período (28 dias) e no segundo período (42 dias) estudado.
Quadro 1. Porcentagem de mortalidade no período posterior a inoculação de Eschertichia coli por via saco aéreo torácico esquerdo

\begin{tabular}{lc}
\hline \multicolumn{1}{c}{ Tratamento } & $\begin{array}{c}\text { Dia 22 a 42Mortalidade \% } \\
\left(\mathrm{n}^{\circ} \text { de aves) }\right.\end{array}$ \\
\hline Controle negativo (T1) & $1,6(2)$ \\
Grupo desafiado (T2) & $48,8(48)$
\end{tabular}

Quadro 2. Porcentagem de lesão no grupo desafiado (T2), no 10 Período (do dia 22 ao dia 28) e no 2o Período (do dia 28 ao dia 42) estudado

\begin{tabular}{|c|c|c|}
\hline Local de lesão & $\begin{array}{c}\text { \% de presença de lesão } \\
\text { no } 1^{\circ} \text { Período }\left(\mathrm{n}^{\circ} \text { de }\right. \\
\left.\text { positivos } / \mathrm{n}^{\circ} \text { de aves }\right)\end{array}$ & $\begin{array}{l}\text { \% de presença de } \\
\text { lesão no } 2^{o} \text { Período } \\
\text { (n }{ }^{\circ} \text { de positivos } / \\
n^{\circ} \text { de aves) }\end{array}$ \\
\hline Saco aéreo torácico direito & $56,18(50 / 89)$ & $66,66(22 / 33)$ \\
\hline Saco aéreo torácico esquerdo & $58,43(52 / 89)$ & $72,72(24 / 33)$ \\
\hline Saco aéreo abdominal & $58,43(52 / 89)$ & $90,90(30 / 33)$ \\
\hline Pericardite & $94,38(84 / 89)$ & $12,12(4 / 33)$ \\
\hline Perihepatite & $39,32(35 / 89)$ & $3,03(1 / 33)$ \\
\hline
\end{tabular}

Quadro 3. Frequência de soros positivos no teste de HI para Mycoplasma gallisepticum nos dois períodos estudados

\begin{tabular}{lcc}
\hline \multirow{2}{*}{ Tratamento } & HI \% de positivos $\left(\mathrm{n}^{\circ}\right.$ de positivo $/ \mathrm{n}^{\circ}$ de aves $)$ \\
\cline { 2 - 3 } & $1^{\text {o }}$ Período & $2^{\circ}$ Período \\
\hline Controle negativo (T1) & $0(0 / 50)$ & $0(0 / 25)$ \\
Grupo desafiado (T2) & $93,33(28 / 30)$ & $75(18 / 24)$
\end{tabular}

\section{DISCUSSÃO}

Os primeiros sinais clínicos foram observados aos 10 dias de experimento quando as aves apresentavam espirro e posteriormente foi detectado edema facial infraorbitário, sendo este um sinal clínico presente em muitas aves. Estes sinais clínicos corroboram aqueles descritos na sinusite infraorbitária por Ley (2008), muito comum em perus.

Também foram observados estertores, secreção nasal e secreção ocular. Estes resultados confirmam os dados obtidos por Bajwa et al. (1992) que inocularam MG em pintos de poedeiras com quatro a cinco dias de idade, por via subcutânea ou intratraqueal. As aves foram infectadas com Eschrichia coli. Os sinais clínicos passaram a ser observados sete dias após a inoculação do MG, ocorreram inicialmente depressão e depois espirros, secreção nasal e estertores. Além disso, verificaram o envolvimento do sistema nervoso e cianose de cristas em algumas aves (Bajwa et al. 1992).

No presente estudo também se observou sinais relacionados ao sistema nervoso em três aves que apresentaram tremores, desequilíbrio e em uma delas também torcicolo e opistótono. Esta sintomatologia pode ter sido causada pelo MG. Chin et al. (1991) relataram três casos de meningoencefalite em perus que foram associadas com MG, as aves eram de diferentes origens, os autores descreveram torcicolo e opistótono como sinais clínicos. As três aves foram positivas para MG na soroaglutinação rápida e HI, no exame histopatológico foi observado moderada a severa encefalite e meningite, em uma das aves o MG foi isolado do cérebro, dos sacos aéreos e da traqueia.

Segundo Nakamura et al. (1994) em um experimento realizado com frangos ocorreu mortalidade de parte das aves vacinadas para doença de Newcastle e Bronquite infeccio- 
sa das galinhas e que foram inoculados por via intranasal com MG e E. coli. Em outro estudo semelhante, mas sem a inoculação de $M G$ não ocorreu mortalidade (Nakamura et al. 1992). Dessa forma, estes resultados demonstraram que MG agrava a infecção por E. coli, aumentando a mortalidade. Um experimento realizado com frangos inoculados com MG e vacinados contra Newcastle mostrou a susceptibilidade a E. coli, sendo esta observada após oito dias da inoculação de MG e da vacina para Newcastle (Gross 1990). Os resultados deste estudo corroboram estas informações.

A cepa F é patogênica para os perus (Lin \& Kleven 1982). Os resultados do presente estudo confirmam a alta patogenicidade da vacina contra MG cepa F para perus. 0 quadro clínico foi semelhante ao observado em infecções naturais por MG. Através da vacinação e consequente desenvolvimento de micoplasmose, as aves se tornaram susceptíveis a E. coli e ocorreu uma alta taxa de mortalidade e presença de lesões como perihepatite, pericardite e aerossaculite.

Bajwa et al. (1992) estudaram a susceptibilidade de aves a E. coli após a infecção por MG e observaram que três diferentes grupos que foram inoculados com $E$. coli na primeira, segunda ou terceira semana após a inoculação com MG tiveram manifestações clínicas graves, mas parecidas entre os grupos, encontraram uma morbidade e mortalidade relativamente alta. 0 grupo que recebeu somente micoplasma apresentou uma mortalidade menor do que os demais e a mortalidade apresentou-se tardia. Esses dados são compatíveis com os resultados encontrados neste estudo, pois a maior mortalidade ocorreu após a inoculação de E. coli.

A transmissão de MG pode ocorrer de forma horizontal, geralmente através de aerossóis (Mettifogo \& Buim 2009). Em 1981, Kleven mostrou que ocorria transmissão horizontal da cepa F de frangos vacinados por via ocular para frangos não vacinados. Em um experimento utilizando frangos se observou a transmissão apenas quando a vacina foi utilizada por via ocular e não quando utilizada como spray. E em consequência à possibilidade de transmissão vertical, a utilização da cepa F é limitada em locais próximos a granja de perus ou de frangos de corte (Evans et al. 2009).

Ley et al. (1993) relataram que entre fevereiro e maio de 1991 alguns lotes de perus na Carolina do Norte apresentaram sinais respiratórios como sinusite, aerossaculite e aumento da mortalidade sendo isolado $M G$. Neste mesmo período o micoplasma foi isolado em um lote de galinhas poedeiras que ficava próximo. Estes autores comprovaram que se tratava da cepa F mostrando a alta transmissão através de aerossóis.

\section{CONCLUSÃO}

A vacina contra MG-F utilizada para poedeiras é patogênica para perus. 0 delineamento experimental utilizado, desafiando as aves no primeiro dia de idade com MG e no $21^{\circ}$ 을 dia com Escherichia coli mostrou-se eficaz para simular a infecção natural por MG e E. coli. Assim, sugere-se que possa ser usado para estudos de sinusite infecciosa em perus, como em testes de medicamentos ou ainda para testes de eficácia de vacinas.

\section{REFERÊNCIAS}

Barnes H.J., Lisa K.N. \& Vaillancourt J.P. 2008. Colibacillosis, p.691-737. In: Saif Y.M., Fadly A.M., Glisson J.R., McDougald L.R., Nolan L.K. \& Swayne D.E. (Eds), Diseases of Poultry. $12^{\text {th }}$ ed. Editora Blackwell Publishing, Ames.

Bajwa N.Z., Siddique M. \& Javed M.T. 1992. Pathogenesis of Escherichia coli in previously Mycoplasma gallisepticum infected layer chickes. J. Islamic Acad. Sci. 5(2):123-126.

Buim M.R., Mettifogo E., Timenetsky J., Kleven S. \& Ferreira A.J.P. 2009. Epidemiological survey on Mycoplasma gallisepticum and M. synoviae by multiplex PCR in commercial poultry. Pesq. Vet. Bras. 29(7):552-556.

Chin R.P., Daft B.M., Meteyer C.U. \& Yamamotoc R. 1991. Meningoencephalitis in commercial meat turkeys associated with Mycoplasma gallisepticum: case report. Avian Dis. 35:986-993.

Evans J.D., Leigh S.A., Branton S.L., Collier S.D., Pharr G.T. \& Bearson S.M.D. 2005. Mycoplasma gallisepticum: Current and developing means to control the avian pathogen. J. Appl. Poult. Res. 14:757-763.

Evans J.D., Branton S.L. \& Leigh S.A. 2009. Effect of dosage and vaccination route on transmission of a live attenuated Mycoplasma gallisepticum vaccine: A broiler model. Avian Dis. 53:416-420.

Garmyn A., Martel A., Froyman R., Nauwynck H., Duchateau L., Haesebrouck F. \& Pasmans F. 2009. Effect of multiple- and single-day enrofloxacin medications against dual experimental infection with avian pneumovirus and Escherichia coli in turkeys. Poult. Sci. 88:2093-2100.

Glisson J.R. 1998. Bacterial respiratory diseases of poultry. Poult. Sci. 77:1139-1142.

Gross W.B. 1990. Factors affecting the development of respiratory disease complex in chickens. Avian Dis. 34(3):607-610.

Kleven S.H. 1981. Transmissibility of the F strain of Mycoplasma gallisepticum in Leghorn chickens. Avian Dis. 25(4):1005-1018.

Kleven S.H. 1998. Mycoplasma in the etiology of Multifactorial Respiratory Disease. Poult. Sci. 77:1146-1149.

Ley D.H., Avakian A.P. \& Berkhoff J.E. 1993. Clinical Mycoplasma gallisepticum infection in multiplier breeder and Meat Turkeys Caused by F Strain: Identification by Sodium Dodecyl Sulfate-Polyacrylamide Gel electrophoresis, restriction endonuclease analysis, and the polymerase chain reaction. Avian Dis. 37:854-862.

Ley D.H. 2008. Mycoplasma gallisepticum infection, p.805-864. In: Saif Y.M., Fadly A.M., Glisson J.R., McDougald L.R., Nolan L.K. \& Swayne D.E. (Eds), Diseases of Poultry. $12^{\text {th }}$ ed. Blackwell Publishing, Ames.

Lin M.Y. \& Kleven S.H. 1982. Pathogenicity of two strains of Mycoplasma gallisepticum in turkeys. Avian Dis. 26(2):360-364.

Mettifogo E. \& Buim M.R. 2009. Mycoplasma gallisepticum, p.86-100. In: Revolledo L. \& Ferreira A.J.P. (Eds), Patologia Aviária. Editora Manole, Barueri, SP.

Nakamura K., Ueda H., Tanimura T. \& Noguchi K. 1994. Effect of mixed live vaccine (Newcastle disease and infectious bronchitis) and Mycoplasma gallisepticum on the chicken respiratory tract and on Escherichia coli infection. J. Comp. Pathol. 111:33-42.

Nakamura K., Narita M., Imai K., Matsumura T., Maeda M. \& Tanimura N. 1992. The effect of mixed live vaccines of Newcastle disease and infectious bronchitis on the chicken respiratory tract. J. Comp. Pathol. 106:341-350. 